Research Paper

\title{
Interleukin 17A promotes gallbladder cancer invasiveness via ERK/NF-KB signal pathway mediated epithelial-to-mesenchymal transition
}

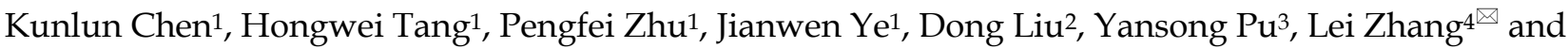 \\ Wenlong Zhai ${ }^{\bowtie}$ \\ 1. Department of Hepatobiliary and Pancreatic Surgery, The First Affiliated Hospital of Zhengzhou University, Zhengzhou, Henan, 450052, China \\ 2. Departments of Gastroenterology, Shaanxi Provincial People's Hospital, Xi'an, Shaanxi 710068, China \\ 3. Departments of General Surgery, Shaanxi Provincial People's Hospital, Xi'an, Shaanxi 710068, China \\ 4. Hepatic Surgery Center, Institute of Hepato-Pancreato-Bililary Surgery, Tongji Hospital, Tongji Medical College, Huazhong University of Science and \\ Technology, Wuhan, 430030, China \\ Kunlun Chen and Hongwei Tang contributed equally.
}

$\square$ Corresponding author: Wenlong Zhai, The First Affiliated Hospital of Zhengzhou University, No 1 Jianshe East Road, Erqi, Zhengzhou, Henan Province, 450052, China, E-mail: fcczhaiwl@zzu.edu.cn; and Lei Zhang, Tongji Hospital, Tongji Medical College, Huazhong University of Science and Technology, Wuhan, 430030, China, E-mail: zhang17803@126.com.

(c) The author(s). This is an open access article distributed under the terms of the Creative Commons Attribution License (https://creativecommons.org/licenses/by/4.0/). See http://ivyspring.com/terms for full terms and conditions.

Received: 2019.09.25; Accepted: 2020.03.27; Published: 2020.05.18

\begin{abstract}
As a pro-inflammatory cytokine, Interleukin 17A (IL-17A) plays an important role in pathology of tumor microenvironment and inflammatory diseases. In this study, we intend to investigate the role of IL-17A on the metastasis of gallbladder cancer (GBC) and related mechanisms. The serum levels of IL-17A were associated with node metastasis and advanced stage. We also found the pro-invasion effect of IL-17A on GBC cells. When treated with IL-17A, the protein level of epithelial marker E-cadherin in GBC cells was significantly down-regulated, while the protein level of the mesenchymal phenotype marker vimentin was significantly increased. IL-17A increased the expression of transcription factor slug, the phosphorylation of ERK $1 / 2$ and the nuclear translocation of NF-KB/p50 and p65 in a concentration-dependent manner. Pretreatment of cells with U0126 could reverse the nuclear translocation of NF-KB/p50 and p65 and EMT induced by IL-17A. IL-17A promotes gallbladder cancer invasiveness via ERK/NF-KB signal pathway mediated epithelial-to-mesenchymal transition. As a new therapeutic targets and diagnostic marker, IL-17A may play an important role in the treatment of GBC.
\end{abstract}

Key words: Interleukin 17A; gallbladder cancer; epithelial-mesenchymal transition; invasiveness; ERK/ nuclear factor- $\mathrm{kB}$ signal pathway

\section{Introduction}

Gallbladder cancer (GBC) has the highest proportion of biliary tract tumors, which causes mass cancer-related death worldwide. The development of gallbladder cancer has been linked to various genetic and environmental factors, and chronic inflammation is the most important risk factor [1,2]. GBC is usually diagnosed at terminal stages owing to the invasive nature and contribution of the non-specific clinical symptom. The 5 -year survival rates were $1 \%, 5 \%$,
$15 \%, 39 \%$ and $60 \%$ for patients with stage IV, stage III, stage II, stage I, and stage $0[3,4]$. It is important to discover mechanisms leading to novel therapeutic targets against GBC.

As a member of IL-17 family, IL-17A was reported to have controversial roles in cancer. Our previous study suggests a promoting effect of IL-17A on tumors [5], whereas other studies suggested its protective role in cancer by enhancing immune 
system-mediated tumor rejection [6]. Metastasis contributes to more than $90 \%$ cancer deaths [1]. The role of IL-17A in the metastasis of GBC is still largely unknown.

In this study, we demonstrated that IL-17A could enhance metastatic capacity of GBC cell via promoting ERK/ NF-kB mediated EMT (epithelial-tomesenchymal transition).

\section{Materials and Methods}

\section{Reagents}

Rabbit poly-clonal antibodies against E-cadherin, rabbit poly-clonal antibody against $\mathrm{N}$-cadherin, rabbit mono-clonal antibody against vimentin, and goat poly-clonal antibody against Snail were purchased from Abcam. Rabbit mono-clonal antibody against Slug was purchased from Cell Signaling. Anti-NF-kB- p65, p50 and Histone H1 antibodies were ordered from Santa Cruz Biotechnology (Santa Cruz, CA, USA). DMEM (Gibco) were used. U0126 were purchased from Sigma.

\section{ELISA}

The serums of blood samples of GBC patients were collected by centrifugation and stored at $80^{\circ} \mathrm{C}$ for subsequent analysis. IL-17A levels were measured by ELISA as previously described [7]. This study was approved by Ethical Committee of the First Affiliated Hospital of Zhengzhou University and in accordance with the Declaration of Helsinki. All patients signed informed consent forms.

\section{Cell culture}

GBC-SD cell line was ordered from the Cell Bank of the Chinese Academy of Sciences (Shanghai, China). GBC-SD cells were cultured in Dulbecco's Modified Eagle's Medium (Gibco) containing fetal bovine serum (10\%) and $100 \quad \mu \mathrm{g} / \mathrm{ml}$ penicillin/streptomycin.

\section{Construction of expression plasmids and transfection}

The construction of the expression plasmids and their transfection were performed as previously described [2]. Briefly, we made the full-length pcDNA3.1 (Invitrogen) MEK1 vector by cloning the full-length PCR product of MEK1 with KOD® DNA polymerase (Toyobo, Osaka, Japan). We used DNA sequencing to confirm the plasmid sequences. For transient transfection experiments, cells were plated $24 \mathrm{~h}$ before transfection in a 6-well plate at a density of $2 \times 10^{5}$ cells per well. For the transfection, we used Lipofectamine 2000 (Invitrogen) with $4.0 \quad \mu g$ pcDNA3.1(+)-MEK1 vector or $4.0 \mu \mathrm{g}$ pcDNA3.1(+) empty vector (as a negative control) in accordance with the manufacturer's protocol.

\section{Cell growth assay}

The colorimetric 3-(4,5-dimethylthiazol-2-yl) 2,5-diphenyltetrazolium bromide assay (MTT) was performed to detect the effect of IL-17A on GBC cells proliferation. In brief, GBC cells $(1 \times 103$ cells/well $)$ were seeded into 96-well plates and treated with IL-17A(R\&D System, Minneapolis, MN) for selected time points, and $20 \mu \mathrm{l}$ of MTT solution $(5 \mathrm{mg} / \mathrm{ml}$; Sigma-Aldrich, St. Louis, MO, USA) was added and then incubated for an additional $4 \mathrm{~h}$ at $37^{\circ} \mathrm{C}$. The

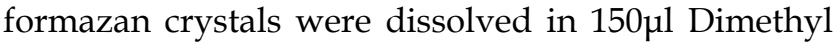
Sulfoxide (DMSO)(Sigma-Aldrich) and the absorbance of samples was measured at $490 \mathrm{~nm}$ using a micro-plate reader (Model 3550; Bio-Rad, Hercules, California, USA). The inhibitory rate of GBC cells proliferation was calculated according to the formula: (1-experimental absorbance value/control absorbance value) $\times 100 \%$. Each experiment was conducted in triplicate.

\section{Cell migration assay}

About $1 \times 10^{6} / \mathrm{ml}$ GBC-SD cells were seeded in 6-well plates and cultured, a vertical long wound was scratched after the cells spread over the plates. GBC-SD cells were cultured with or without IL-17A (50 ng/ml) (R\&D System, Minneapolis, MN) for $48 \mathrm{~h}$, then image was captured. The wound closure ratio was calculated as follows: $(0 \mathrm{~h}$ width- $48 \mathrm{~h}$ wound width) $/ 0 \mathrm{~h}$ wound width.

\section{Cell invasion assay}

Invasion assay was measured using 24-well BioCoat Matrigel Invasion Chambers(Becton Dicknson, Bedford, MA) as described previously[8]. After pretreated with IL-17A for $24 \mathrm{~h}$, cells were added into inner well and cultured for $24 \mathrm{~h}$, cells that did not invade were wiped off and then cells that invaded through the matrigel was fixed with formalin, dyed with crystal violet and measured by counting the cell number under 5 microscopic vision fields.

\section{Western blotting analysis}

Whole cell lysates were extracted with radioimmunoprecipitation assay buffer (R\&D System, Minneapolis, MN) on ice for $20 \mathrm{~min}$. Nuclear lysates were extracted as previously mentioned [5]. Cells were suspended in $100 \mu \mathrm{l}$ of lysis buffer. After separating on $10 \%$ SDS-polyacrylamide gel electrophoresis, proteins were transferred onto PVDF membranes. The membranes were subsequently blocked in defatted milk to reduce non-specific binding at $37^{\circ} \mathrm{C}$ for $1 \mathrm{~h}$ and then incubated with different antibodies (E-cadherin, $\mathrm{N}$-cadherin, vimentin, Snail, Slug, Histone H1, ERK, p-ERK, 
NF-kB-p50, p65or beta-actin).

The bands were measured with an enhanced chemiluminescence kit (Millipore, Billerica, MA, USA) and exposed by autoradiography. The densitometric analysis was carried out with Image J software (GE Healthcare, Buckinghamshire, UK).

\section{Statistical analysis}

All data are presented as the mean \pm standard deviation. Differences between groups were assessed using the Student's t-test followed by the Shapiro-Wilk W test or one-way analysis of variance followed by Bonferroni's test. $\mathrm{P}<0.05$ was considered to indicate a statistically significant difference. Analyses were performed using SPSS 17.0 software (SPSS Inc., Chicago, IL, USA).

\section{Results}

\section{The serum concentrations of IL-17A was related with the clinicopathological features of the GBC cases}

Patients with lymph node metastasis have higher serum concentrations of IL-17A, compared with those without node metastasis. Patients with advanced stage (III-IV) also have higher serum concentrations of IL-17A. The serum concentrations of IL-17A was associated node metastasis and advanced stages (Table 1).

\section{IL-17A increases cell motility in GBC cells}

We found that IL-17A had no effect on the apoptosis and proliferation of GBC-SD cells (Figure
1A and 1B). As indicated in Figure 1C and 1D, the wound closure areas in the IL-17A groups were large, respectively. The pro-migration effect of IL-17A was also verified using chambers with matrigel. Treatment with IL-17A markedly enhanced the migration ability of cells. Furthermore, as shown in Figure 1E and 1F, IL-17A increased the number of cells that pass through the membrane significantly, suggesting the pro-invasion effect of IL-17A. In conclusion, IL-17A could enhance the ability of migration and invasion of GBC cells.

Table 1. Association between Serum levels of IL-17A and the clinicopathological features of the GBC cases.

\begin{tabular}{llll}
\hline Variables & No. of cases & $\begin{array}{l}\text { Serum levels of IL-17A } \\
(\mathrm{pg} / \mathrm{ml})\end{array}$ & P-value \\
\hline $\begin{array}{l}\text { Age (years) } \\
<60\end{array}$ & 10 & 7.4020 & 0.981 \\
$\geq 60$ & 24 & 7.4554 & \\
Sex & 17 & 7.6894 & 0.804 \\
Female & 17 & 7.1900 & \\
Male & & & \\
Histological grade & 24 & 7.1283 & 0.631 \\
Well and moderate & 10 & 8.1870 & \\
Poor & & & 0.025 \\
N status & 21 & 5.7395 & 0.803 \\
N0 & 13 & 10.162 & \\
N1/2 & & & \\
Tumor size (cm) & 23 & 7.6126 & \\
$<5$ & 11 & 7.0782 & \\
$\geq 5$ & & & \\
Clinical stage & 9 & 2.9689 & \\
I-II & 25 & 9.0492 & \\
III-IV & & & \\
\hline
\end{tabular}

A

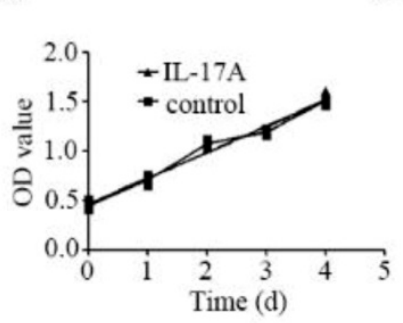

$\mathrm{D}$

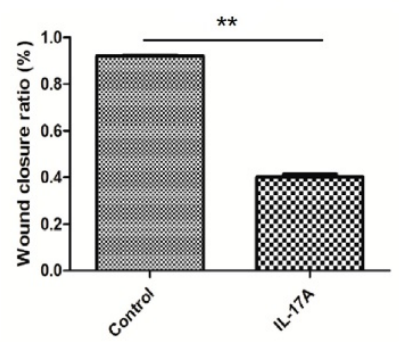

$\mathrm{B}$

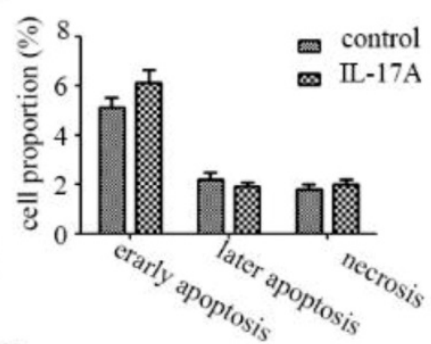

E

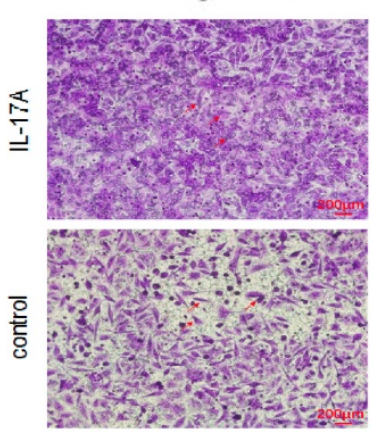

$\mathrm{C}$

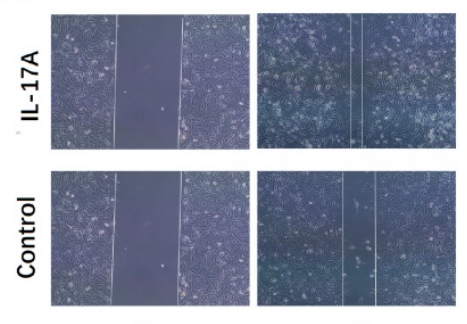

$\mathrm{F}$

0
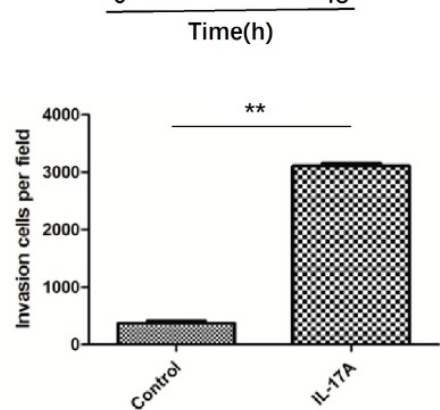

Figure 1. The pro-metastasis effect of IL-17A in GBC. (A) IL-17A has no effect on the proliferation of GBC cells. (B) IL-17A has no effect on the apoptosis of GBC cells. (C and D) The effect of IL-17A on cell migration was detected by using wound healing assays. IL-17A enhanced the motility of GBC cells. (E) The pro-invasion effect of IL-17A on GBC cells was assay via transwell assay. (F) The invasion cells per field were calculated. All data are presented as the mean \pm standard deviation. *p $<0.05$ was considered to indicate a statistically significant difference. 
A

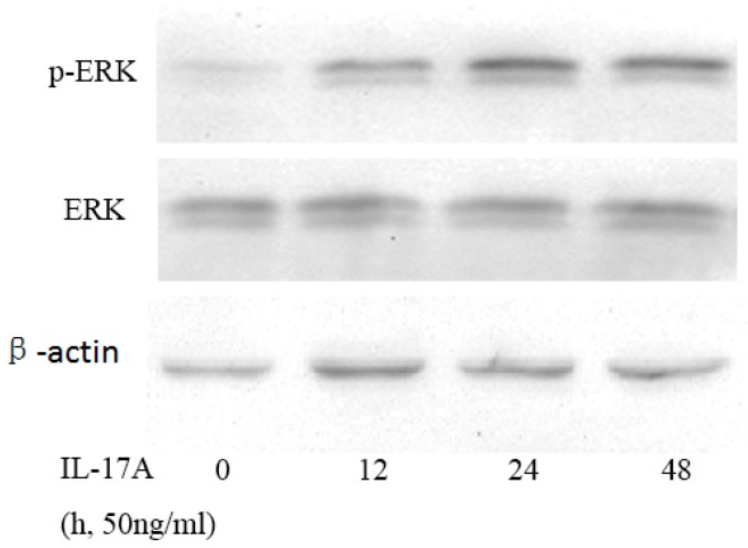

$\mathrm{B}$

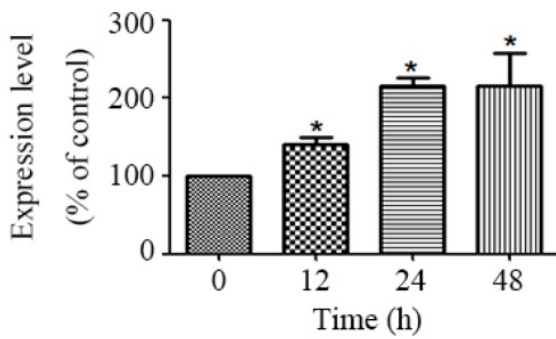

Figure 2. IL-17A activates ERK signal pathway in GBC cells. (A)The protein level of p-ERK and ERK was analysed with Western blotting analysis in GBC cells treated with IL-17A $(50 \mathrm{ng} / \mathrm{ml})$ at different time points. (B) Phosphorylation densities of ERK were digitally scanned. All data are presented as the mean \pm standard deviation. $* p<0.05$ was considered to indicate a statistically significant difference.

\section{IL-17A activates the activity of ERK signal pathway in GBC cells}

We detected the effect of IL-17A on the activity of ERK signal pathway in GBC cells. The phosphorylation of ERK1/2, which represents the activity of ERK signal pathway, was significantly up-regulated by IL-17A in a time depend manner (Figure 2). To further verify the specific effect of baicalein on ERK pathway, we transfected GBC cells with a plasmid (pcDNA3.1(+)-MEK1) expressing human MEK1 (Figure S1) and found that MEK1 enhanced the pro-invasion effect of IL-17A on cell invasion (Figure S2).

\section{IL-17A regulates EMT marker and related transcription factor expressions in GBC cells}

The expression of epithelial marker E-cadherin in IL-17A treated group cells was significantly down-regulated (Figure 3A and 3B), while the expression of the mesenchymal phenotype markers vimentin was significantly increased. The expression of transcription factor slug can also be up-regulated by IL-17A (Figure 3A and 3B).

\section{IL-17A promotes the nuclear translocation of NF-KB}

To further clarify the role of NF-KB transactivation in the pro-invasion effect of IL-17A, we detect the expression levels of transcription factors in GBC cells treated with IL-17A. We found that IL-17A significantly induced the nuclear translocation of NF-kB/ p65 and p50 (Figure 3C and 3D).

\section{IL-17A regulates EMT via activating ERK signal pathway}

After treated with an ERK inhibitor (U0126; 10 $\mu \mathrm{M})$ for $30 \mathrm{~min}, \mathrm{GBC}$ cells were cultured with IL-17A or not to explore the potential relationships between ERK signal pathway, NF-KB and EMT markers. Pretreated with U0126 $(10 \mu \mathrm{M})$, the expression levels of vimentin were significantly decreased, the expression levels of E-cadherin were significantly increased (Figure 4C-D) compared with IL-17A treated cells. The study clarified that IL-17A induced EMT in GBC cell via activating ERK signal pathway. Pretreated with U0126 could block the nuclear translocation of NF-kB/p50 and p65. Accordingly, the pro-invasion effect of IL-17A in GBC cells could also been partially reversed by U0126 (Figure 4A and B). In conclusion, our results show that IL-17A up-regulates the activity of ERK signal pathway, then induces the nuclear translocation of NF-kB/p50 and p65, subsequently induces $\mathrm{EMT}$, and promotes the pro-invasion effect in GBC cells at last.

\section{Discussion}

Relationship between inflammation and tumorigenesis is well known, chronic infection has been found to be associated with GBC formation, but the contribution is still unclear and requires more investigation [9]. Because of its aggressive factor, GBC is usually diagnosed at terminal stages, the prognosis is poor [10]. In our previous study, we found a pro-invasion effect of IL-17A in esophageal adenocarcinoma cells [5]. Our results show that IL-17A enhanced the invasion of GBC cells without effecting the proliferation and apoptosis. IL-17A up-regulated the activity of ERK signal pathway, then promoted the nuclear translocation of NF-KB and promoted EMT at last.

GBC is related with chronic inflammatory diseases closely, in which various inflammatory cytokines are detected. IL-17A plays crucial roles in the occurrence and development of lots of 
inflammatory diseases and is also frequently found in cancer immunity and microenvironment $[5,11]$. In the study, we clarified that the serum levels of IL-17A were associated with lymphatic metastasis and advanced stages. The results were similar to previous research, in which IL17 producing TCR $\delta(+)$ in peripheral blood was related to poor prognosis of GBC patients[12], IL-17+ cells in the tumor cooperatively facilitated pathogenesis and progression of GBC [13]. But all the results are from retrospective clinical study, the molecular mechanisms of IL-17 in the development and prognosis of GBC need to be further explored.

Our results showed the pro-invasion effect of IL-17A in GBC cells. EMT could enhance the ability of movement and deformation of cancer cells, further cause the spread of cancer cells. Acquisition of mesenchymal markers, loss of epithelial markers and dysfunction of EMT-related transcription factors have been detected and are related with the prognosis and clinicopathology of GBC patients [11]. The reduction of the protein level of E-cadherin is related with loss of adhesive mechanisms of GBC and CD8+ T cell infiltration at invasive areas of GBC $[14,15]$. Compare with primary tumors, the expression of vimentin is higher in metastases and is related with GBC lymphatic metastasis and metastasis [16]. Slug, a EMT-related transcription factor, is one of the events that influence the regulation of EMT in GBC cells [17]. To further exploit the mechanism of the pro-invasion effect of IL-17A on GBC cell, we detected the effect of IL-17A on cell EMT. In the study, we found that IL-17A could significantly up-regulate the protein level of vimentin and slug, and down-regulated the protein level of E-cadherin. Therefore, the pro-invasion effect of IL-17A on GBC is associated with inducing EMT.

Accumulating evidence suggests the cooperation of signal pathways take part in the stepwise EMT regulatory network in GBC, including ERK [18] and NF-kB [19]. The over-activity of ERK signal pathway is found in both GBC tissues and cell lines [20]. As a key regulator of the invasion of gallbladder cancer [21], IL-17A exerts its effect via regulating NF-kB in various cell lines [5]. So we investigated whether the induction of EMT by IL-17A is via regulating the ERK
A
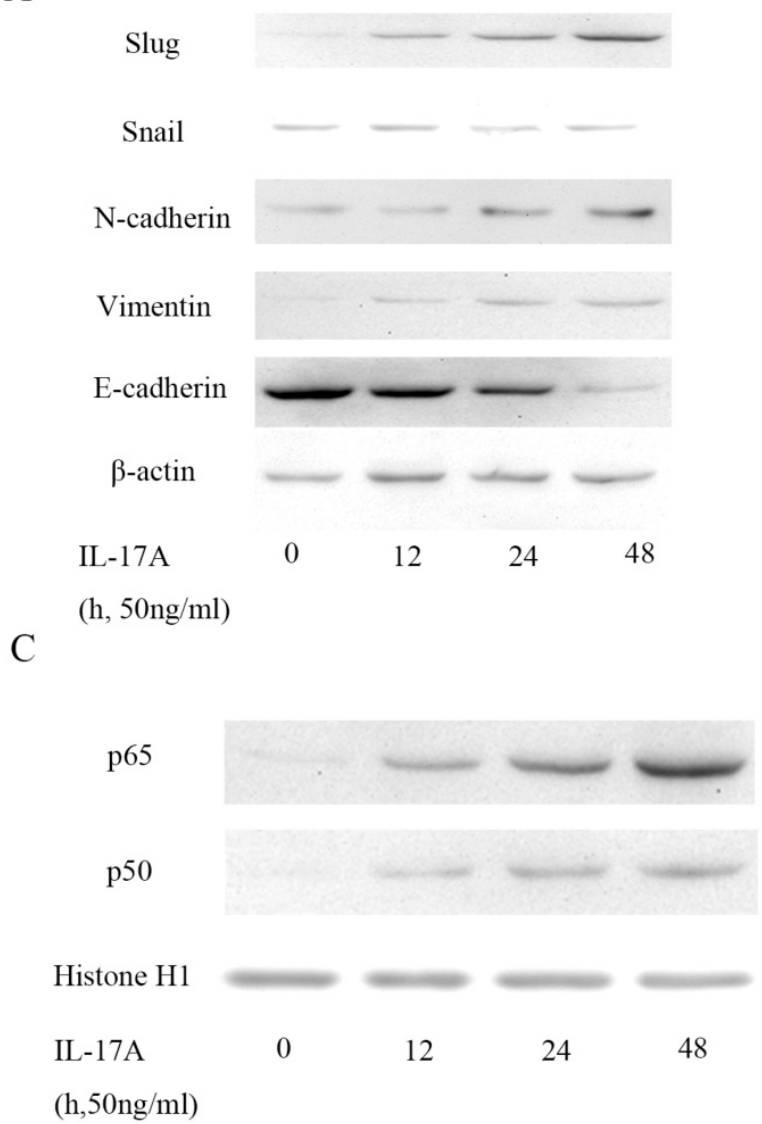

B

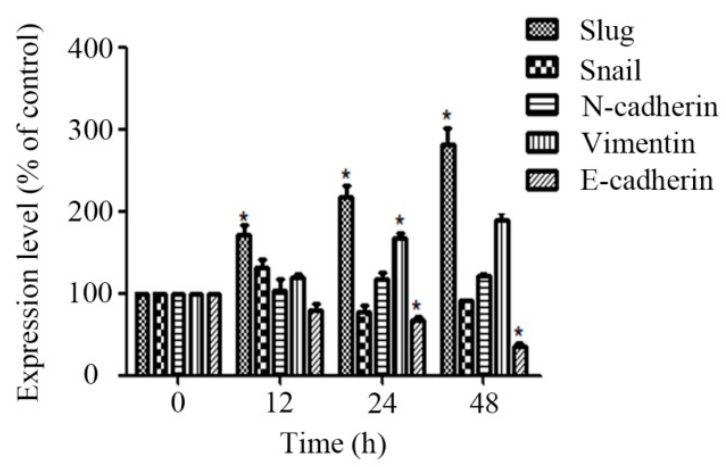

$\mathrm{D}$

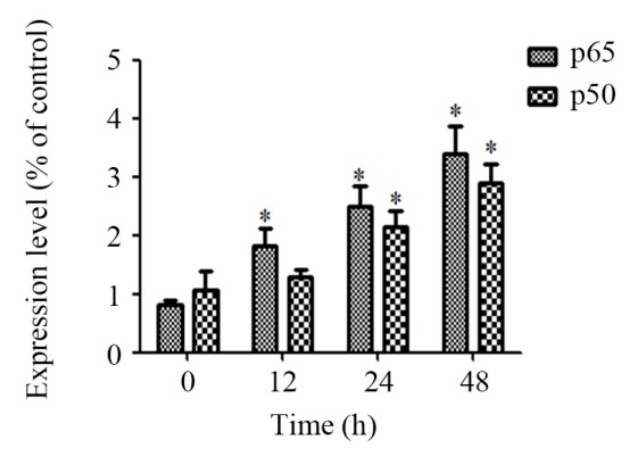

Figure 3. IL-17A induces EMT of GBC cells. (A) Protein levels of Snail, E-cadherin, N-cadherin, Vimentin and Slug in GBC cells treated with IL-17A (50 ng/ml) for $24 \mathrm{~h}$ or not were detected with western blot. (B) The expression levels of E-cadherin, Vimentin, N-cadherin, Snail and Slug were quantified as percentage of control. (C) The effect of IL-17A on protein expressions of NF-KB/p50 and p65. (D) Quantification of the expressions of NF-KB/p50 and p65. All data are presented as the mean \pm standard deviation. ${ }^{*} p<0.05$ was considered to indicate a statistically significant difference. 
and NF-kB signal pathway. In the study, we found that IL-17A enhanced the activity of ERK signal pathway and subsequently nuclear translocation of the p65 and p50 subunits of GBC cells. The pro-invasion effect could be partly reversed by ERK inhibitor, suggesting that ERK/NF-KB signal pathway played an important role in the induction of EMT by IL-17A on GBC cells. In conclusion, our results showed that IL-17A could enhance the ability of migration and invasion of GBC cells via inducing EMT via regulating ERK/NF-KB signal pathway. Further characterization of the pro-invasion effect of
IL-17A may help us to find therapeutic targets and diagnostic marker in future.

\section{Supplementary Material}

Supplementary figures.

http://www.jcancer.org/v11p4406s1.pdf

\section{Acknowledgments}

The project was supported by National Natural Science Foundation of China (No. 81702863).
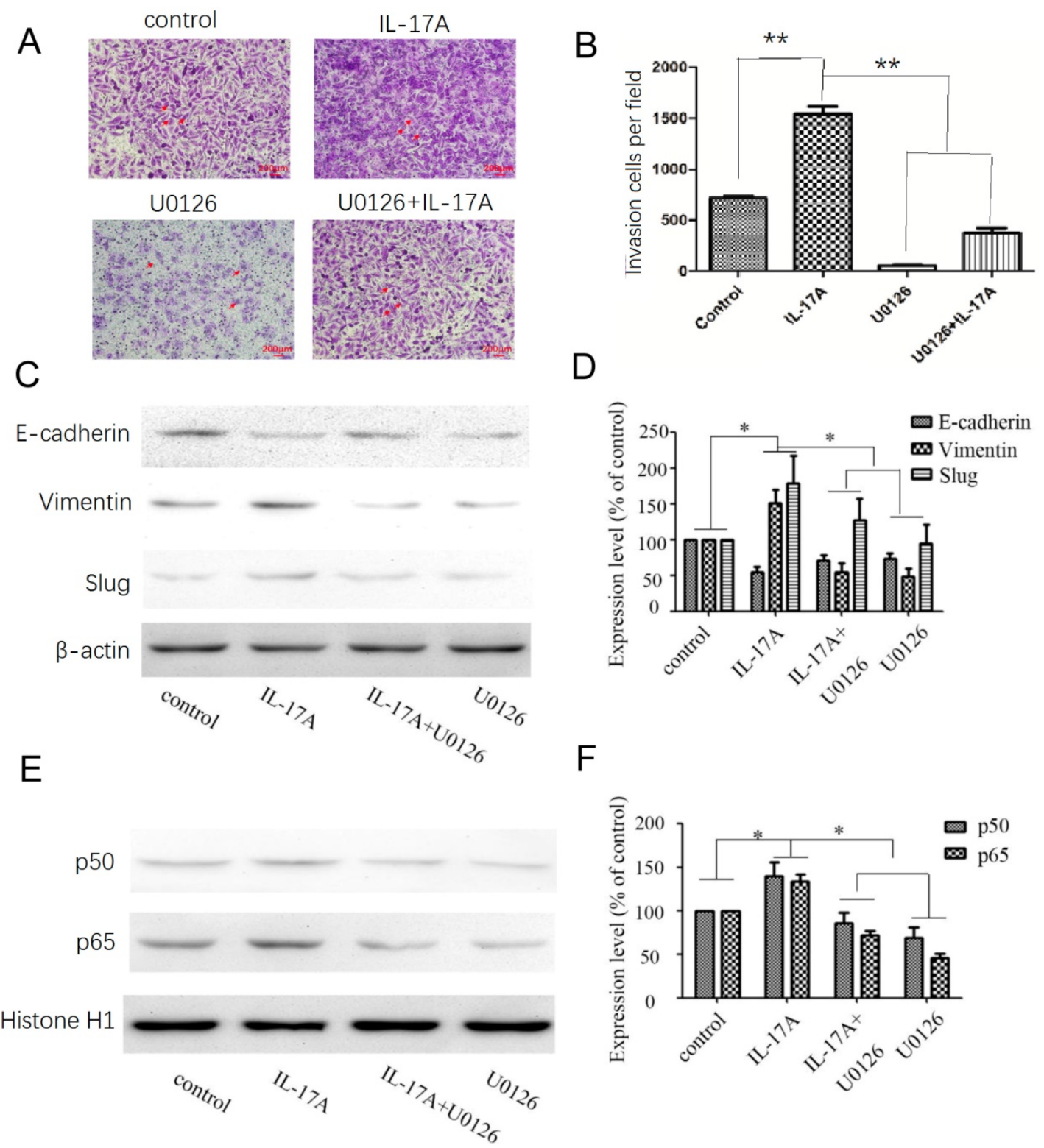

$\mathrm{F}$

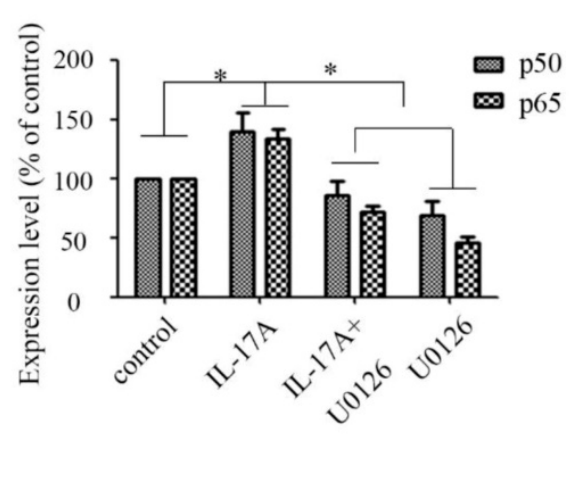

Figure 4. Effects of the ERK signal pathway inhibitor and IL-17A on cell invasion and EMT in GBC cells. (A, B) The pro-invasion effect of IL-17A in GBC cells could been partially reversed by U0126. (C, D) After treated with an ERK inhibitor (U0126; $10 \mu M)$ for 30 min, GBC cells were cultured with IL-17A or not. Pretreated with U0126 (10 $\mu M)$, the expression levels of Vimentin were significantly decreased, the expression levels of E-cadherin were significantly increased. (E, F) Pretreated with $U 0126$ could block the nuclear translocation of NF-KB/p50 and p 65 in GBC cells. All data are presented as the mean \pm standard deviation. $* p<0.05$ was considered to indicate a statistically significant difference. 


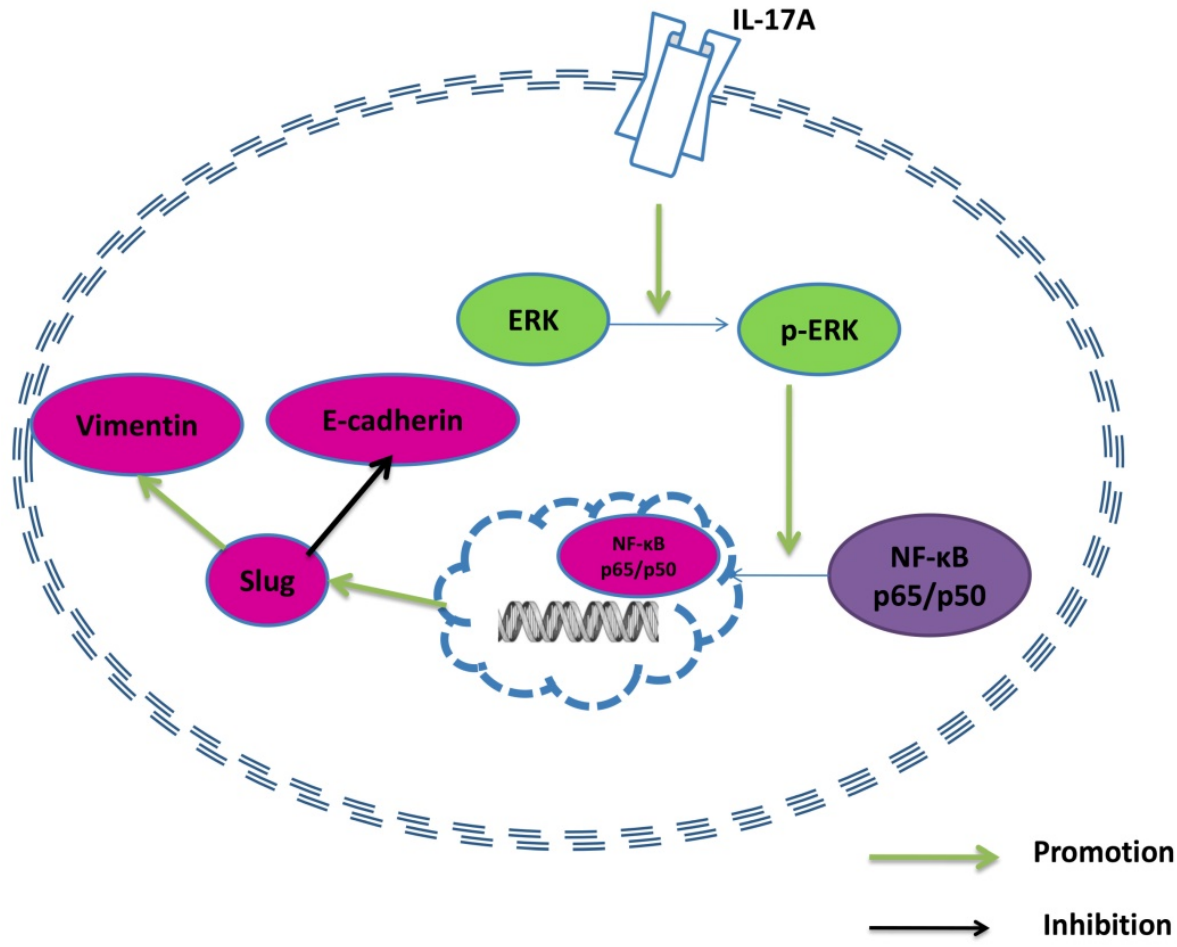

Figure 5. A model of the effect of IL-17A in GBC cells.

\section{Competing Interests}

The authors have declared that no competing interest exists.

\section{References}

1. Sheth S, Bedford A, Chopra S. Primary gallbladder cancer: recognition of risk factors and the role of prophylactic cholecystectomy. Am J Gastroenterol. 2000;95:1402-10

2. Tazuma S, Kajiyama G. Carcinogenesis of malignant lesions of the gall bladder. The impact of chronic inflammation and gallstones. Langenbecks Arch Surg. 2001; 386: 224-9.

3. Donohue JH, Stewart AK, Menck HR. The National Cancer Data Base report on carcinoma of the gallbladder, 1989-1995. Cancer. 1998;83(12):2618-28.

4. Duffy A, Capanu M, Abou-Alfa GK, Huitzil D, Jarnagin W, Fong Y, et al. Gallbladder cancer (GBC): 10-year experience at Memorial Sloan-Kettering Cancer Centre (MSKCC). J Surg Oncol. 2008; 98: 485-9.

5. Liu D, Zhang $R, W u$ J, Pu Y, Yin $X$, Cheng $Y$, et al. Interleukin-17A promotes esophageal adenocarcinoma cell invasiveness through ROS-dependent, NF-kappaB-mediated MMP-2/9 activation. Oncol Rep. 2017; 37: 1779-85.

6. Hus I, Bojarska-Junak A, Chocholska S, Tomczak W, Wos J, Dmoszynska A, et al. Th17/IL-17A might play a protective role in chronic lymphocytic leukemia immunity. PLoS One. 2013; 8: e78091.

7. Pu Y, Zhang S, Zhou R, Huang N, Li H, Wei W, et al. IL-17A up-regulates expression of endothelial tissue factor in liver cirrhosis via the ROS/p38 signal pathway. Biochem Biophys Res Commun. 2016; 470: 41-7.

8. Chen K, Zhang S, Ji Y, Li J, An P, Ren H, et al. Baicalein inhibits the invasion and metastatic capabilities of hepatocellular carcinoma cells via down-regulation of the ERK pathway. PLoS One. 2013; 8: e72927.

9. Wang R, Yang L, Zhang C, Wang R, Zhang Z, He Q, et al. Th17 cell-derived IL-17A promoted tumor progression via STAT3/NF-kappaB/Notch1 signaling in non-small cell lung cancer. Oncoimmunology. 2018; 7: e1461303.

10. Ye J, Chen K, Qi L, Li R, Tang H, Zhou C, et al. Metformin suppresses hypoxiainduced migration via the HIF1alpha/VEGF pathway in gallbladder cancer in vitro and in vivo. Oncol Rep. 2018; 40: 3501-10.

11. Xu S, Zhan M, Wang J. Epithelial-to-mesenchymal transition in gallbladder cancer: from clinical evidence to cellular regulatory networks. Cell Death Discov. 2017; 3: 17069.

12. Patil RS, Shah SU, Shrikhande SV, Goel M, Dikshit RP, Chiplunkar SV. IL17 producing gammadeltaT cells induce angiogenesis and are associated with poor survival in gallbladder cancer patients. Int J Cancer. 2016; 139: 869-81.

13. Zhang Y, Huang Y, Qin M. Tumour-infiltrating FoxP3+ and IL-17-producing T cells affect the progression and prognosis of gallbladder carcinoma after surgery. Scand J Immunol. 2013; 78: 516-22.
14. Puhalla $\mathrm{H}$, Herberger $\mathrm{B}$, Soleiman $\mathrm{A}$, et al. E-cadherin and beta-catenin expression in normal, inflamed and cancerous gallbladder tissue. Anticancer Res. 2005;25:4249-54

15. Kai K, Masuda M, Aishima S. Inverse correlation between CD8(+) inflammatory cells and E-cadherin expression in gallbladder cancer: Tissue microarray and imaging analysis. World J Clin Cases. 2017; 5: 1-8.

16. Dong $\mathrm{P}, \mathrm{He} \mathrm{XW}, \mathrm{Gu}$, et al. Vimentin significantly promoted gallbladder carcinoma metastasis. Chin Med J (Engl). 2011;124:4236-44.

17. Lee DG, Lee SH, Kim JS, Park J, Cho YL, Kim KS, et al. Loss of NDRG2 promotes epithelial-mesenchymal transition of gallbladder carcinoma cells through MMP-19-mediated Slug expression. J Hepatol. 2015; 63: 1429-39.

18. Zhang R, Ma M, Lin XH, Liu HH, Chen J, Chen J, et al. Extracellular matrix collagen I promotes the tumor progression of residual hepatocellular carcinoma after heat treatment. BMC Cancer. 2018; 18: 901.

19. Varghese E, Samuel SM, Abotaleb M, Cheema S, Mamtani R, Busselberg D. The "Yin and Yang" of Natural Compounds in Anticancer Therapy of Triple-Negative Breast Cancers. Cancers (Basel). 2018; 10.

20. Buchegger $K$, Silva R, Lopez J, Ili C, Araya JC, Leal P, et al. The ERK/MAPK pathway is overexpressed and activated in gallbladder cancer. Pathol Res Pract. 2017; 213: 476-82

21. Kong $\mathrm{X}, \mathrm{Ma} \mathrm{MZ}$, Zhang $\mathrm{Y}$, et al. Differentiation therapy: sesamin as an effective agent in targeting cancer stem-like side population cells of human gallbladder carcinoma. BMC Complement Altern Med. 2014; 254:1186-1472 